Classification

Physics Abstracts

9.210

\title{
ÉVAPORATION DU SÉLÉNIURE DE ZINC EN TUBE OUVERT SOUS FLUX D'ARGON ET D'HYDROGËNE
}

\author{
J. CHEVRIER, D. ETIENNE et G. BOUGNOT \\ Centre d'Etudes d'Electronique des Solides (Associé CNRS) \\ Université des Sciences et Techniques du Languedoc \\ place E.-Bataillon, 34060 Montpellier, France
}

(Reçu le 22 juin 1973)

\begin{abstract}
Résumé. - Nous nous sommes intéressés au phénomène d'évaporation d'un solide sous flux gazeux dans les domaines de simple diffusion et de diffusion-convection. Le transport en phase vapeur du séléniure de zinc, sous flux d'argon ou d'hydrogène, a été ainsi décrit et l'on montre que, dans le domaine de vitesse étudié, le régime d'équilibre thermodynamique, associé à la loi de Fick suffit à rendre compte des résultats expérimentaux.

Abstract. - We have been interested in thermodynamical equilibria describing the transport in vapor phase of a zinc selenide source exposed to an argon or hydrogen flow, at high temperature. We have studied the evaporation of that compound in the low linear velocity range. Then, we have shown the influence of hydrogen with respect to the inert gas and confirmed the use of the most recent thermochemical data for $\mathrm{SeH}_{2}(\mathrm{~g})$ which account well for our experimental results. Fick's diffusion law has been successfully applied to these results.
\end{abstract}

1. Introduction. - Le transport dans un tube ouvert d'un composé solide, d'une source chaude vers une source froide, par l'intermédiaire d'une phase vapeur, s'effectue généralement dans les conditions pratiques suivantes : dans une enceinte cylindrique, on impose le long de l'axe un profil thermique schématisé sur la figure 1 . Une source plane, placée dans la zone chaude, à une température $T_{1}$, est balayée par un mélange gazeux, chimiquement neutre ou actif, dont la vitesse linéaire est définie, à l'entrée, par

$$
v_{0}=\frac{d_{0}}{S} \text { en } \mathrm{cm} \cdot \mathrm{s}^{-1}
$$

où

$d_{0}$ est le débit gazeux mesuré à l'entrée en $\mathrm{cm}^{3} \cdot \mathrm{s}^{-1}$, $S$ la section de l'enceinte en $\mathrm{cm}^{2}$.

A une distance $L$ où est imposée une température $T_{1}^{\prime}$, est, en général, positionné un substrat monocristallin favorisant une croissance épitaxiale du composé provenant de la source; les taux de croissance, dans ce type de fabrication, sont limités généralement par le transfert de masse en phase vapeur, à la source, plutôt que par les cinétiques d'interface : Klosse et Ullersma [1] ont abordé théoriquement cet aspect ; nous nous limiterons quant à nous au phénomène de transfert lui-même, dans le cas du séléniure de zinc.

Expérimentalement, on détermine la perte de poids

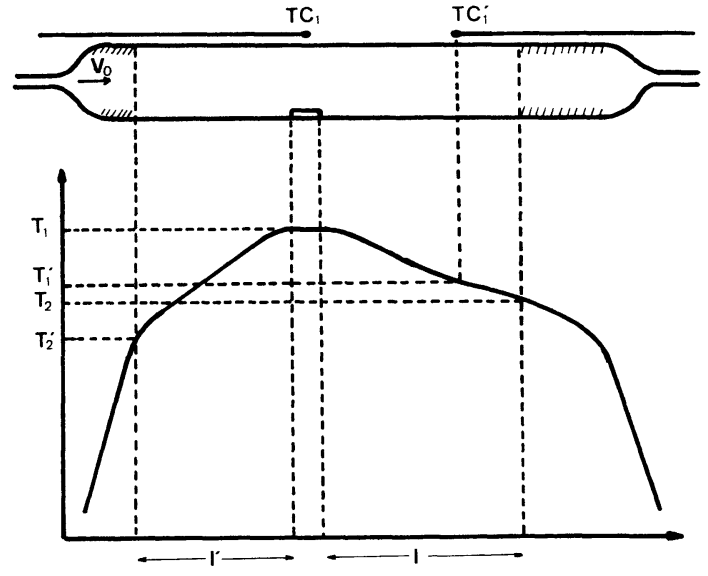

Fig. 1. - Profil thermique type d'une manipulation de transport en tube ouvert.

$Q(t)$ de la source après une durée $t$ de transport et l'on en tire un taux d'évaporation massique en régime permanent « $G »$ tel que :

$$
\frac{Q(t)}{t}=G=J_{\mathrm{E}} A \text { en } \mathrm{g} \cdot \mathrm{h}^{-1}
$$

où

$J_{\mathrm{E}}$ est le flux d'évaporation massique en $\mathrm{g} \cdot \mathrm{cm}^{-2} \cdot \mathrm{h}^{-1}$

$A$ la surface d'évaporation en $\mathrm{cm}^{2}$. 
D'une façon générale, on considère [2], [3] que le taux d'évaporation réactive, dans le cas d'une réaction chimique superficielle, irréversible et du premier ordre, entre le milieu gazeux et le solide, varie avec la vitesse linéaire du courant gazeux suivant la courbe de la figure 2 .

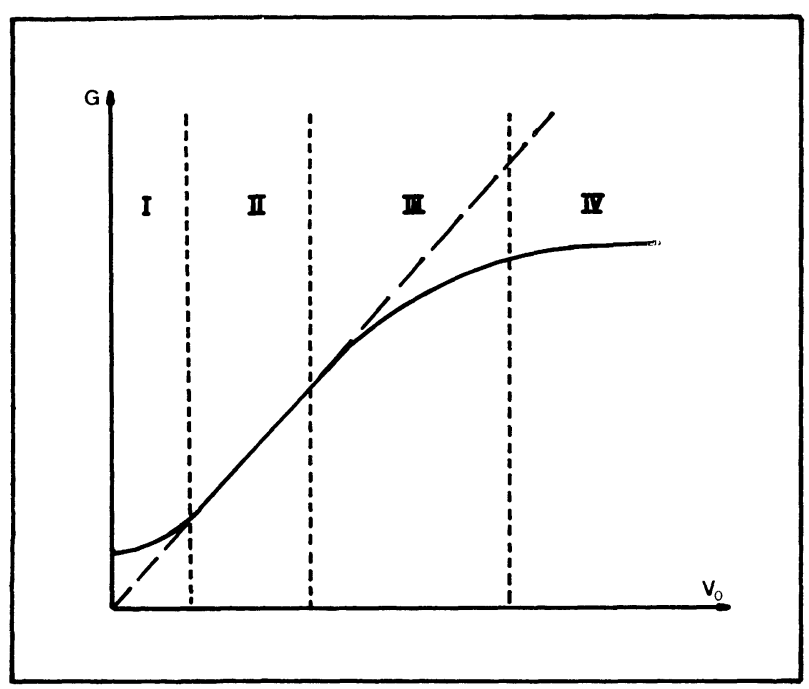

FIG. 2. - Variation du taux d'évaporation réactive en fonction de la vitesse linéaire $v_{0}$ du gaz vecteur.

On distingue ainsi quatre régimes de transport :

- dans les régimes I et II, la vitesse d'entraînement est assez faible pour que l'équilibre thermodynamique ait le temps de s'établir en tous points de la phase vapeur ; la composition de celle-ci est donc uniforme en zone chaude, constante au cours du temps et égale à la composition d'équilibre à la température $T_{1}$ :

a) dans la zone I, le transport de la vapeur vers la zone froide est entièrement contrôlé par la diffusion due à la différence de température $T_{1}-T_{2}=D T$, et à la rétrodiffusion due à la différence de température $T_{1}-T_{2}^{\prime}=D T^{\prime}$

b) dans la zone II, l'entraînement du mélange gazeux est purement convectif ;

c) dans les régimes III et IV, la vitesse d'entraînement est trop élevée pour que l'équilibre thermodynamique soit réalisé en tous points de la phase vapeur. C'est le domaine de formation d'une couche limite (zone III) puis d'un transport contrôlé par les réactions en surface (zone IV).

2. Appareillage utilisé. - Nous avons décrit dans un précédent article [4] le type d'appareillage utilisé. La source est positionnée dans une enceinte en quartz cylindrique, de $30 \mathrm{~mm}$ de diamètre, en position la plus basse possible. Le $\mathrm{ZnSe}$, de pureté $5 \mathrm{~N}$, est comprimé de façon à présenter au flux gazeux une surface plane rectangulaire de $6 \mathrm{~cm}^{2}$. La source est conservée dans l'enceinte, mais en zone froide, pendant la mise en température du four et ce n'est qu'après stabilisation qu'elle est introduite lentement grâce à un système étanche de translation; on élimine ainsi le régime transitoire. Par cette méthode, l'erreur commise sur la mesure des taux d'évaporation, compte tenu de la mise en température de la source depuis l'ambiante, a été évaluée à moins de $2 \%$ pour des manipulations de plusieurs heures.

Deux thermocouples de régulation $\left(\mathrm{TC}_{1}\right.$ et $\left.\mathrm{TC}_{1}^{\prime}\right)$ imposent la différence $T_{1}-T_{1}^{\prime}$ définie précédemment.

3. Résultats expérimentaux. - Les résultats expérimentaux concernant les taux d'évaporation, pour des vitesses d'entraînement de 0 à $1 \mathrm{~cm} . \mathrm{s}^{-1}$, sont donnés sur les figures 3 et 4 pour diverses températures de source. On y distingue effectivement les régimes de transport I, II et III ; cependant la région II est pratiquement inexistante pour $\mathrm{ZnSe}$ transporté à l'argon. Nous nous sommes limités ici à l'étude des régimes diffusifs et convectifs. C'est en effet ce domaine qui se prête le mieux à la comparaison avec la théorie comme l'ont montré les études effectuées sur GaAs et $\mathrm{GaP}$ [5].

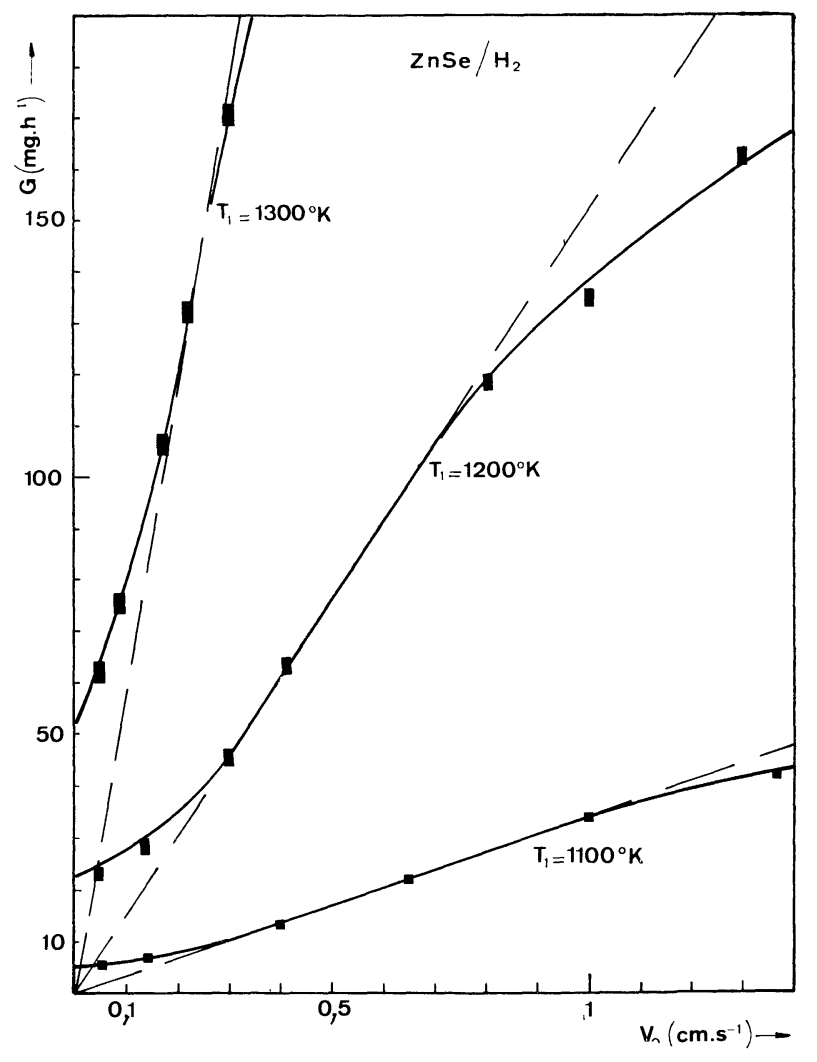

FIG. 3. - Variation expérimentale des taux d'évaporation du ZnSe en fonction de $v_{0}$, dans le cas du transport à l'hydrogène pour $\Delta T=50\left(\Delta T=T_{1}-T_{1}^{\prime}\right)$.

Il est indispensable, dans un premier temps, de déterminer les pressions partielles d'équilibre des espèces gazeuses comportant $\mathrm{Zn}$ ou Se. Ces pressions partielles fixent les concentrations en zone chaude et en zone froide, donc les conditions aux limites. 


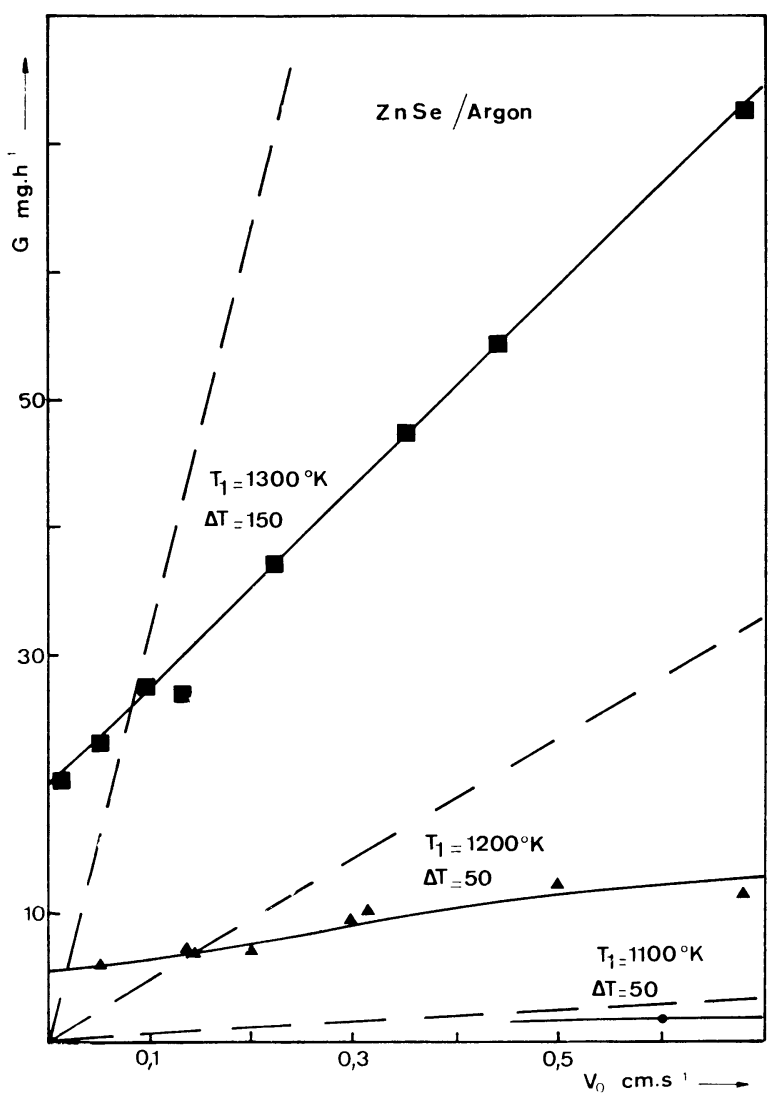

FIG. 4. - Variation expérimentale des taux d'évaporation du $\mathrm{ZnSe}$ en fonction de $v_{0}$, dans le cas du transport à l'argon.

4. Détermination des pressions partielles d'équilibre des espèces chimiques intervenant dans le transport. Cette détermination est fondée sur le principe de calcul proposé par Hurle et Mullin [6].

4.1 Transport A L'ARgON. - La sublimation du $\mathrm{ZnSe}$, ainsi que la mesure des pressions partielles en résultant ont fait l'objet de nombreuses publications et ont permis d'établir certaines données thermochimiques de base : enthalpie standard $\left(\mathrm{H}_{298}^{\mathrm{o}}\right)$, entropie standard $\left(\mathrm{S}_{298}^{\mathrm{o}}\right)$, chaleur spécifique à pression constante $\left(c_{\mathrm{p}}\right)$. Pour ce type de composé, on peut admettre une dissociation à haute température basée sur l'équilibre :

$$
2 \mathrm{ZnSe}(\mathrm{s}) \rightleftharpoons 2 \mathrm{Zn}(\mathrm{g})+\mathrm{Se}_{2}(\mathrm{~g}) \text {. }
$$

Il est possible d'introduire d'autres espèces, issues essentiellement du sélénium; en effet, la vapeur de sélénium comporterait en quantité non négligeable des molécules de $\mathrm{Se}_{6}$ et $\mathrm{Se}_{5}$ [7], [8].

Nous avons considéré seulement un équilibre supplémentaire, à savoir :

$$
3 \mathrm{Se}_{2}(\mathrm{~g}) \rightleftharpoons \mathrm{Se}_{6}(\mathrm{~g}) \text {. }
$$

En admettant la stœchiométrie respectée tant en phase vapeur qu'en phase solide, il vient :

$$
p_{\mathrm{Zn}}=2 p_{\mathrm{Se}_{2}}+6 p_{\mathrm{Se}_{6}}
$$

où les " $p_{i}$ » correspondent aux pressions partielles d'équilibre. Les données thermodynamiques du $\mathrm{ZnSe}$ sont rassemblées dans le tableau I. La seule valeur, couramment admise, de la chaleur spécifique $c_{\mathrm{p}}$, pour le $\mathrm{ZnSe}$, est due à Goldfinger-Jeunehomme [9].

\section{TABLEAU I}

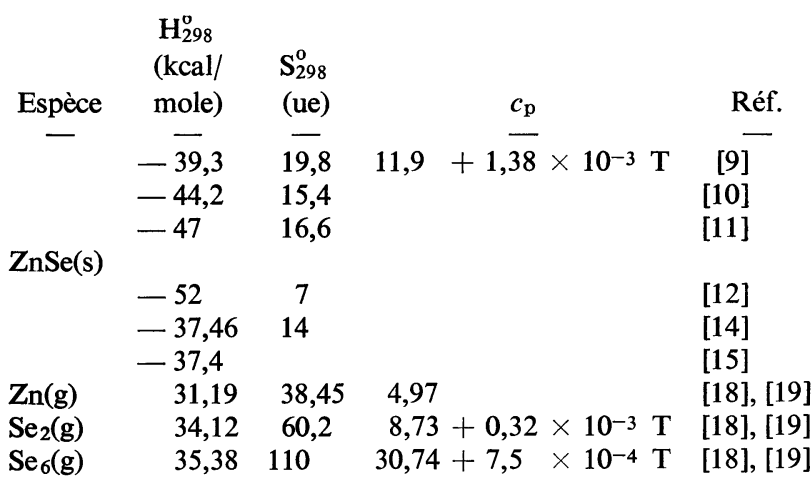

Selon la méthode de Hurle et Mullin, on peut tracer un réseau de courbes représentant les variations des pressions partielles de chaque espèce avec la température (Fig. 5). L'ambiguïté portant essentiellement

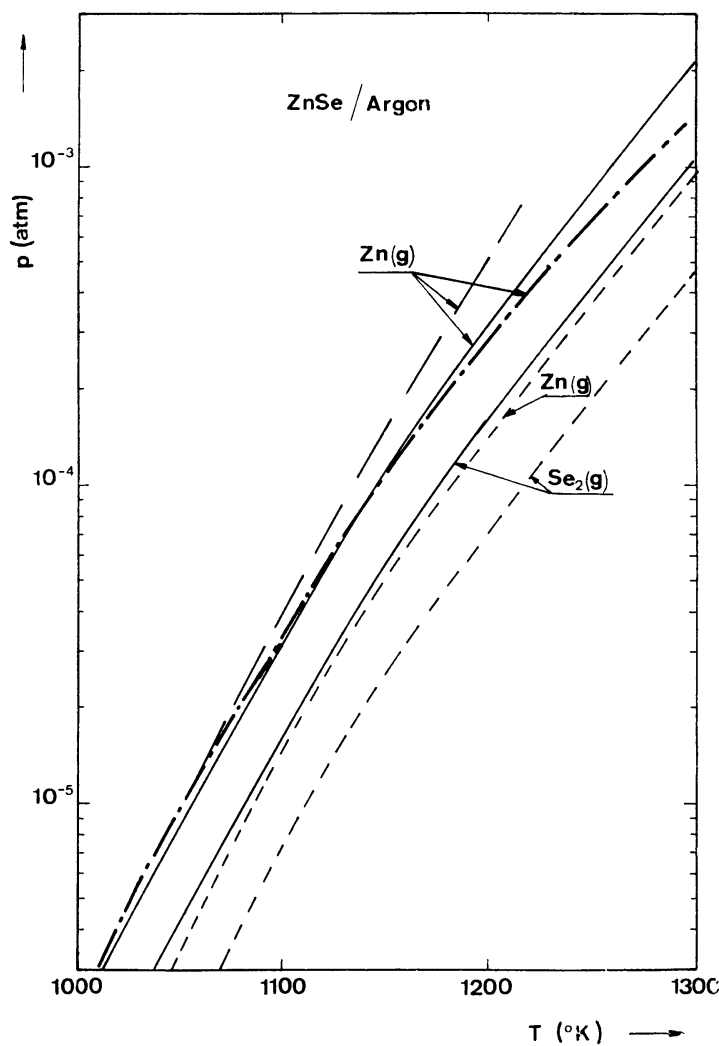

FIG. 5. - Variations des pressions partielles d'équilibre des espèces gazeuses, intervenant dans le transport du $\mathrm{ZnSe}$ sous argon, en fonction de la température :

$$
\begin{aligned}
& \text { - _ - d'après Korneeva } \\
& \ldots \ldots \text { d'après Wösten-Geers } \\
& \hline \ldots \text { nos calculs, d'après Sedwick-Agule } \\
& \text { - nos calculs, d'après Goldfinger. }
\end{aligned}
$$


sur les données du $\mathrm{ZnSe}$, nous avons représenté un réseau calculé à partir des enthalpie et entropie standards de Goldfinger et un autre issu de celles de Sedwick-Agule [10].

La comparaison des valeurs que nous trouvons avec d'autres citées dans la littérature [11], [12] est bonne, compte tenu des incertitudes de base. Nous retiendrons donc les données thermodynamiques de SedgwickAgule, comme l'ont fait récemment encore, Yim et Stofko [13] dans une étude analogue.

4.2. TRANSPORT A L'HYDROGÈNE. - La comparaison des figures 3 et 4 montre que le taux d'évaporation sous flux d'hydrogène est nettement supérieur au taux d'évaporation sous flux d'argon, dans les mêmes conditions de géométrie et de gradient. L'hydrogène ne sert donc pas uniquement de gaz vecteur, il doit présenter une réactivité chimique non négligeable avec la source de $\mathrm{ZnSe}$ solide. Notons que Lilley, Jones et Litting ont fait des constatations semblables dans le transport du sulfure de zinc sous flux d'argon [16] ou d'hydrogène [17].

Les équilibres chimiques que l'on considère ici sont alors les suivants :

$$
\begin{aligned}
\left.2 \mathrm{ZnSe}_{\mathrm{s}}\right) & \rightleftharpoons 2 \mathrm{Zn}(\mathrm{g})+\mathrm{Se}_{2}(\mathrm{~g}) \\
3 \mathrm{Se}_{2}(\mathrm{~g}) & \rightleftharpoons \mathrm{Se}_{6}(\mathrm{~g}) \\
\mathrm{H}_{2}(\mathrm{~g})+\frac{1}{2} \mathrm{Se}_{2}(\mathrm{~g}) & \rightleftharpoons \mathrm{SeH}_{2}(\mathrm{~g})
\end{aligned}
$$

correspondant à l'équilibre principal

$$
\mathrm{ZnSe}(\mathrm{s})+\mathrm{H}_{2}(\mathrm{~g}) \rightleftharpoons \mathrm{Zn}(\mathrm{g})+\mathrm{SeH}_{2}(\mathrm{~g})
$$

dont tiennent compte les quelques auteurs s'intéressant au même genre de problème. Nous supposerons là encore que la stœchiométrie est respectée, ce qui se traduit par l'équation :

$$
\frac{n_{\mathrm{Zn}}-n_{\mathrm{Se}}}{n_{\mathrm{H}}}=0
$$

les $n_{i}$ étant les nombres d'atomes de l'espèce $i$ par unité de volume : la pression totale sera considérée ici comme une variable indépendante :

$$
p_{0}=\sum_{i} p_{i}
$$

Les données thermodynamiques de l'hydrogène étant bien connues, le tableau II donne les diverses valeurs concernant $\mathrm{SeH}_{2}(\mathrm{~g})$ trouvées dans la littérature.

La figure 6 montre les trois réseaux de courbes décrivant les variations des pressions partielles avec la température obtenue avec les couples de données $\mathrm{A}$, $\mathrm{B}$ et $\mathrm{C}$ du tableau II.

\section{TABLEAU II}

$\begin{array}{cccc} & \mathrm{H}_{298}^{\mathbf{0}}(\mathrm{kcal} / \mathrm{mole}) & \mathrm{S}_{298}^{\text {o }}(\mathrm{ue}) & \text { Réf. } \\ \mathrm{A} & 20,50 & \overline{52,9} & {[18],[21]} \\ \mathrm{B} & 9,174 & 55,9 & {[22],[13]} \\ \mathrm{C} & 8 & 53 & {[23],[24]}\end{array}$

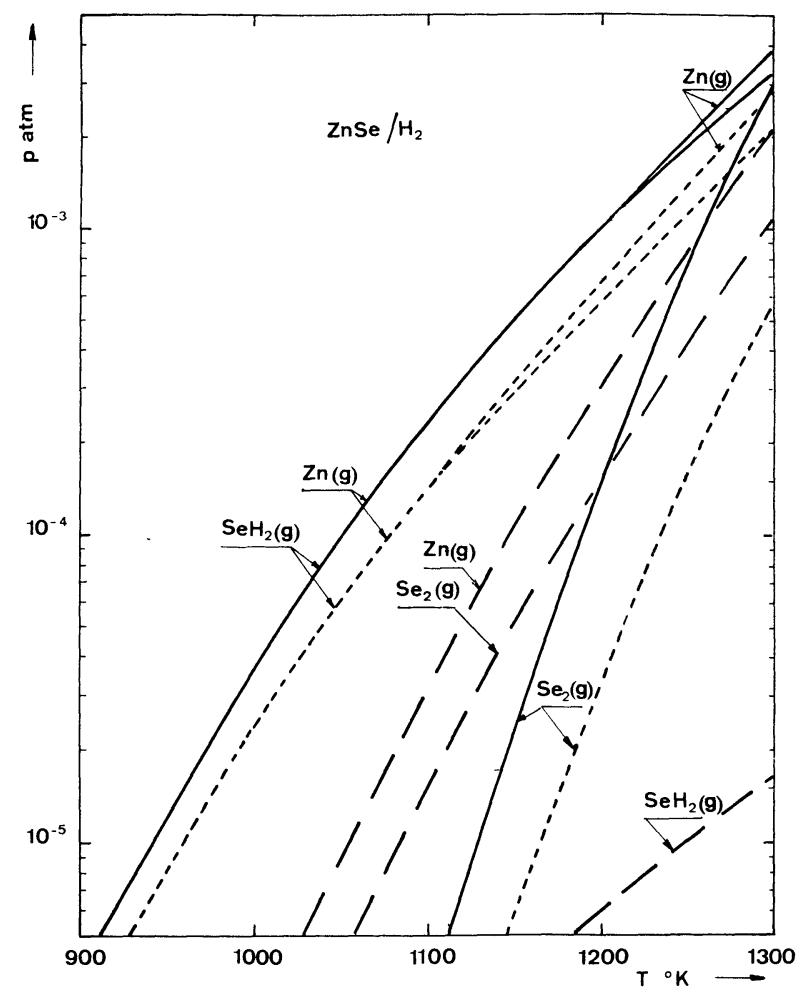

Fig. 6. - Variations des pressions partielles d'équilibre des espèces gazeuses, intervenant dans le transport du ZnSe sous hydrogène, en fonction de la température, pour trois couples de données $\left(\mathrm{H}_{298}^{0}, \mathbf{S}_{298}^{0}\right)$ du $\mathrm{SeH}_{2}(\mathrm{~g})$

$$
\left.\begin{array}{c}
\ldots \ldots \text { données A } \\
-\ldots \text { données B } \\
-\ldots \text { données C }
\end{array}\right\} \text { (tableau II). }
$$

Dans le cas $\mathrm{A}$, le transport du sélénium est essentiellement assuré par l'espèce $\mathrm{Se}_{2}$, l'espèce $\mathrm{SeH}_{2}$ n'intervenant qu'à basse température ; les pressions partielles trouvées sont du même ordre de grandeur que celles obtenues avec l'argon : cela correspondrait à une faible réactivité de l'hydrogène. Dans les deux autres cas, ce transport est assuré par $\mathrm{SeH}_{2}$ dont la pression est nettement supérieure à celle du $\mathrm{Se}_{2}$. L'imprécision sur les données de base est donc très grande, mais nous pourrons fixer notre choix sur les données $\mathbf{B}$ qui, sont plus récentes que les données $\mathrm{C}$, bien que toutes deux rendent bien compte de la réactivité de l'hydrogène par rapport au gaz neutre en faisant apparaître l'espèce $\mathrm{SeH}_{2}$. Cet effet aurait pu être attribué à l'existence possible d'un hydrure de zinc, or l'espèce $\mathrm{ZnH}_{2}(\mathrm{~g})$ se décompose dès $90^{\circ} \mathrm{C}$ [25] et ses données thermochimiques ont été peu étudiées. Seules existent pour l'espèce $\mathrm{ZnH}(\mathrm{g})$ les valeurs recommandées par Kelley [19], [20]. L'introduction d'un équilibre supplémentaire

$$
\frac{1}{2} \mathrm{H}_{2}(\mathrm{~g})+\mathrm{Zn}(\mathrm{g})=\mathrm{ZnH}(\mathrm{g})
$$

ne modifie pas les pressions déjà trouvées dans les trois systèmes précédents. Le tableau III donne les valeurs calculées des pressions partielles de $\mathrm{ZnH}(\mathrm{g})$; 
TABleAU III

$\begin{array}{ccccccc}T(\mathrm{~K}) & 800 & 900 & 1000 & 1100 & 1200 & 1300 \\ p_{\mathrm{ZnH}}(\overline{\mathrm{atm}}) & 0,7 \times 10^{-14} & 0,56 \times 10^{-12} & 0,18 \times 10^{-10} & 0,32 \times 10^{-9} & 0,33 \times 10^{-8} & 0,257 \times 10^{-7}\end{array}$

elles sont effectivement négligeables dans le domaine de température étudié et ne pourraient pas justifier l'utilisation des données A.

5. Taux d'évaporation. Comparaison avec la théorie. - Aux faibles vitesses (régions I et II), l'évacuation du mélange gazeux, en équilibre avec la source dans la zone chaude, s'effectue par diffusion convective de part et d'autre de la source. En régime permanent, le flux massique dans le sens du courant gazeux est donné par

$$
J_{\mathrm{DC}}=\frac{-\Delta c}{1-\exp \left(\frac{v l}{D}\right)} v+c_{1} v
$$

et le flux de rétrodiffusion par

$$
J_{\mathrm{RC}}^{\prime \prime}=\frac{-\Delta c^{\prime}}{1-\exp \left(-\frac{v l^{\prime}}{D^{\prime}}\right)} v+c_{1} v
$$

$v$ est la vitesse linéaire d'entraînement définie à la température $T$ par

$$
v=\frac{v_{0} T}{T_{0}}
$$

$l$ et $l^{\prime}$ sont les longueurs de diffusion que nous avons assimilées aux distances entre les extrémités de la source et les positions des dépôts en zones froides sur. les parois de l'enceinte (voir Fig. 1) ; $D$ et $D^{\prime}$ sont les coefficients de diffusion moyen entre la source et les zones de dépôts

$$
\begin{aligned}
\Delta c & =c_{1}-c_{2} \\
\Delta c^{\prime} & =c_{1}-c_{2}^{\prime}
\end{aligned}
$$

où $c_{1}, c_{2}$ et $c_{2}^{\prime}$ sont les concentrations d'équilibre aux températures $T_{1}, T_{2}$ et $T_{2}^{\prime}$ d'une espèce gazeuse fictive ZnSe.

Conformément au modèle de Fergusson et Gabor

$$
p_{\mathrm{ZnSe}}(T)=p_{\mathrm{Zn}}(T) \text {. }
$$

Les concentrations s'obtiennent à partir des pressions partielles données sur les figures 5 et 6 , en supposant les gaz parfaits, grâce à la relation :

$$
c_{\mathrm{ZnSe}}(T)=\frac{p_{\mathrm{ZnSe}}(T)}{R T} M_{\mathrm{ZnSe}}
$$

$M_{\mathrm{ZnSe}}$ étant la masse molaire du $\mathrm{ZnSe}$.

On suppose ainsi que l'équilibre thermodynamique est réalisé en tous points de l'enceinte et en particulier que la condensation en zones froides y impose la concentration d'équilibre.

Le taux d'évaporation massique est alors donné par

$$
G=J_{\mathrm{E}} A=\left(\left|J_{\mathrm{DC}}\right|+\left|J_{\mathrm{RC}}\right|\right) S .
$$

Cette formule peut être développée sous la forme

$$
\begin{aligned}
G=\frac{M S V}{R}\left\{\frac{p_{1}}{T_{1}}\left[\frac{\exp \left(\frac{-v l^{\prime}}{D^{\prime}}\right)}{1-\exp \left(\frac{v l^{\prime}}{D^{\prime}}\right)}-\frac{\exp \left(\frac{v l}{D}\right)}{1-\exp \left(\frac{v l}{D}\right)}\right]+\right. \\
\left.+\frac{p_{2}}{T_{2}}\left[\frac{1}{1-\exp \left(\frac{v l}{D}\right)}\right]-\frac{p_{2}^{\prime}}{T_{2}^{\prime}}\left[\frac{1}{1-\exp \left(\frac{v l^{\prime}}{D^{\prime}}\right)}\right]\right\}
\end{aligned}
$$

la limite de $G$ lorsque $v$ tend vers zéro s'écrit

$$
G_{0}=\frac{M S}{R}\left\{\left(\frac{p_{1}}{T_{1}}-\frac{p_{2}}{T_{2}}\right) \frac{D}{l}+\left(\frac{p_{1}}{T_{1}}-\frac{p_{2}^{\prime}}{T_{2}^{\prime}}\right) \frac{D^{\prime}}{l^{\prime}}\right\} .
$$

A grande vitesse, la rétrodiffusion s'annule

$$
\text { (mathématiquement pour } v=\frac{D^{\prime}}{l^{\prime}} \ln \frac{c_{1}}{c_{2}} \text { ) }
$$

et

$$
G=\frac{M S}{R} \frac{p_{1}}{T_{1}} v
$$

ce qui donne bien une variation linéaire de $G$ avec $v$ correspondant au régime II défini précédemment.

Sur les figures 3 et 4 , on peut comparer nos résultats expérimentaux avec la partie linéaire de l'expression théorique de $G$. Dans la zone de régime II, l'accord est excellent en ce qui concerne l'évaporation sous hydrogène. Cette concordance justifie $a$ posteriori l'utilisation des données $\mathrm{B}$ pour le $\mathrm{SeH}_{2}(\mathrm{~g})$. L'inexistence de cette zone, dans le cas de l'évaporation sous argon, permet seulement de conclure que, pour une simple sublimation, sans réaction avec le gaz porteur, on passe sans transition du régime purement diffusionnel au régime de couche limite : le transport doit être alors mieux décrit par des théories de dynamique des fluides pour lesquelles existent des variations de $G$ en $v^{1 / 2}$ [26].

Nous avons ici mis en équation le seul cas du transport réactif à faible vitesse.

Sur les figures 7 et 8 ont été agrandies les gammes des basses vitesses. On y a reporté les valeurs des flux de diffusion $S J_{\mathrm{DC}}$ et de rétrodiffusion $S\left|J_{\mathrm{RC}}\right|$, calculées à partir des valeurs de $l$ et de $l^{\prime}$ relevées expérimenta- 


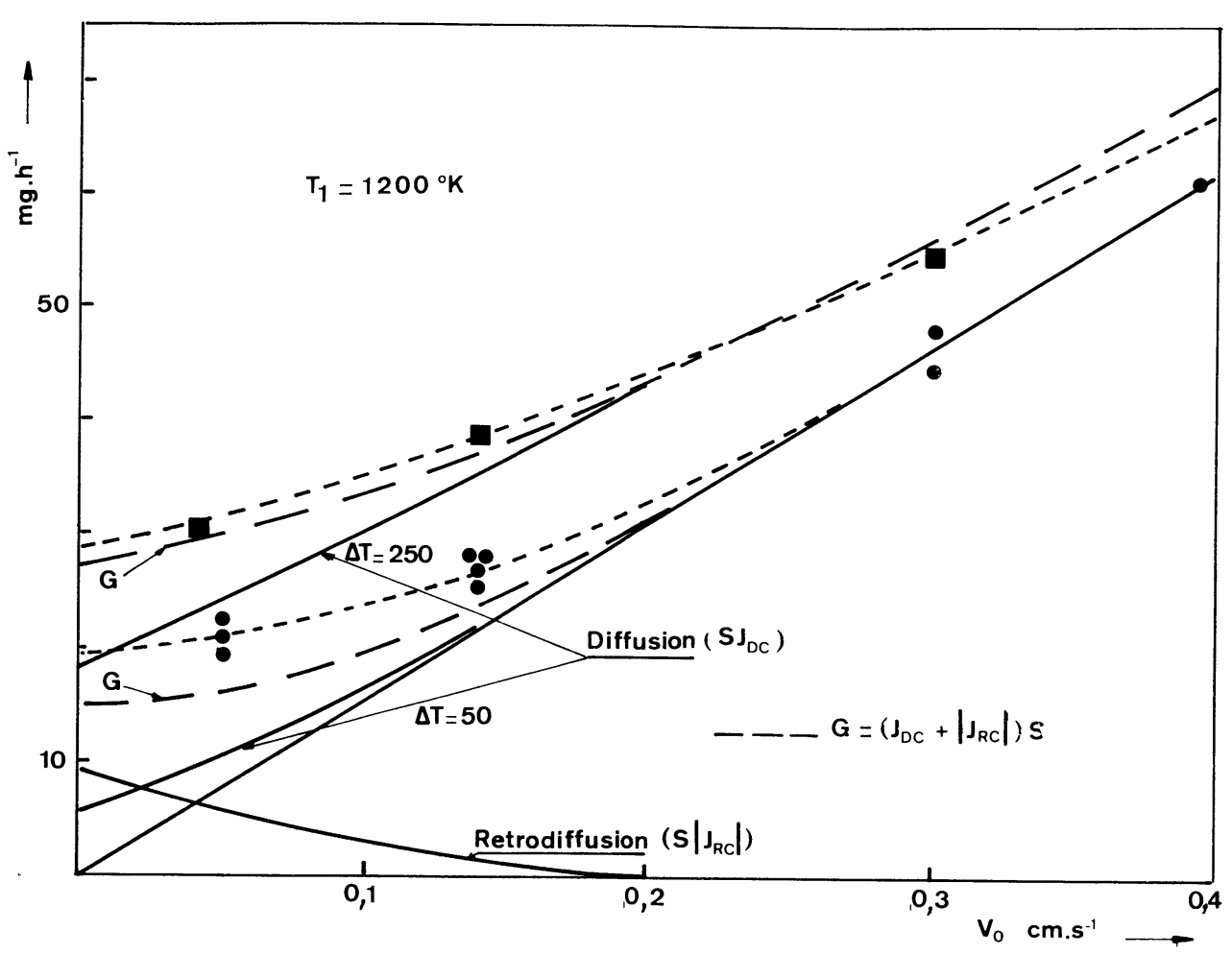

FIG. 7. - Taux d'évaporation du ZnSe sous l'hydrogène dans le domaine des basses vitesses pour $T_{1}=1200 \mathrm{~K}$ :

a) $\Delta T=50: l=37 \mathrm{~cm}, T_{2}=1000 \mathrm{~K}, l^{\prime}=22 \mathrm{~cm}$,

b) $\left.\Delta T=250: l=5,5 \mathrm{~cm}, T_{2}=1160 \mathrm{~K}\right\} \quad T_{2}^{\prime}=1000 \mathrm{~K}$.

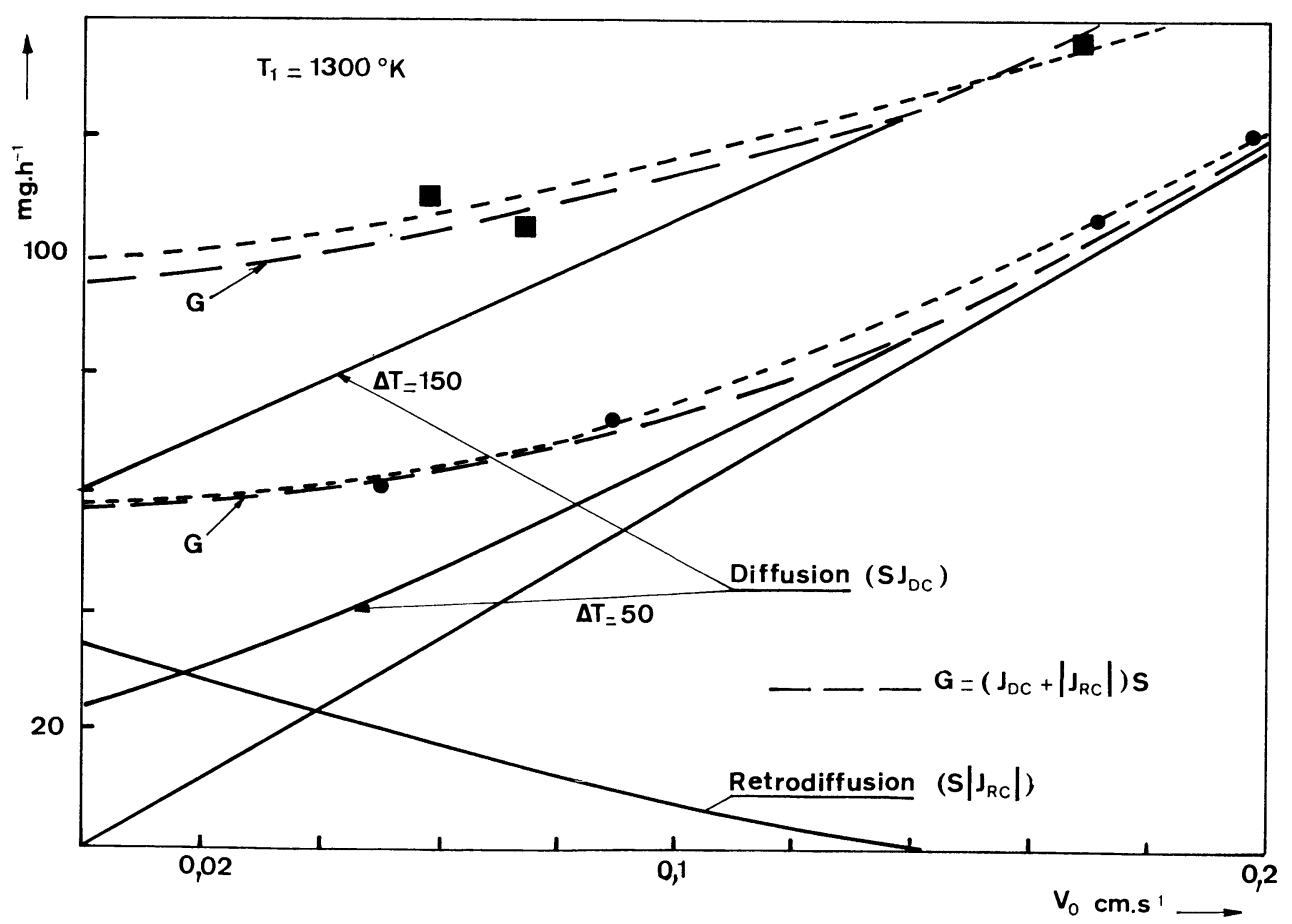

FIG. 8. - Taux d'évaporation du ZnSe sous hydrogène dans le domaine des basses vitesses pour $T_{1}=1300 \mathrm{~K}$ :

a) $\Delta T=50: l=33 \mathrm{~cm}, T_{2}=1140 \mathrm{~K}, l^{\prime}=22 \mathrm{~cm}$,

b) $\left.\Delta T=150: l=8 \mathrm{~cm}, T_{2}=1240 \mathrm{~K}\right\} \quad T_{2}^{\prime}=1140 \mathrm{~K}$. 
lement ainsi que des valeurs de $T_{2}$ et $T_{2}^{\prime}$ en découlant. Pour une température de source $\left(T_{1}\right)$ donnée, la rétrodiffusion garde une valeur ne dépendant que de $v_{0}$, alors que la diffusion dépend en plus du gradient $\Delta T\left(T_{1}-T_{1}^{\prime}\right)$; dans tous les cas, la somme des deux flux et la courbe expérimentale correspondante se recoupent bien et tendent, lorsque $v_{0}$ augmente, vers la limite "convective» représentée là encore par la droite d'équilibre thermodynamique.

Plusieurs expressions théoriques nous permettaient de calculer les coefficients de diffusion binaire des espèces à base de zinc et de sélénium dans l'hydrogène ; quelles qu'elles soient, elles nous ont montré que l'espèce $\mathrm{SeH}_{2}(\mathrm{~g})$ diffusait moins vite que $\mathrm{Zn}(\mathrm{g})$ : nous avons alors admis que c'était cette espèce qui contrôlait le transport de $\mathrm{ZnSe}$ et c'est la valeur de son coefficient de diffusion en fonction de $T$ qui a été retenue. La formule de calcul des coefficients $D$ et $D^{\prime}$ proposée par Hirschfelder [27] a été préférée à celle, plus élaborée, de Wilke et Lee [28] dans la mesure où elle nous a permis un meilleur ajustement des courbes théoriques sur l'expérience. L'accord en résultant a été trouvé très satisfaisant et justifie l'influence de la rétrodiffusion et celle du gradient thermique $\left(T_{1}-T_{1}^{\prime}\right)$ sur le transfert de matière en phase vapeur.

6. Conclusion. - A partir d'expériences d'évaporation en tube ouvert et par comparaison avec un modèle d'équilibre simple, nous avons justifié certaines données thermochimiques de la littérature ainsi que l'utilisation d'une loi simple de diffusion. Cette justification, par une telle méthode, ne peut être qu'approchée mais l'accord obtenu s'est révélé très correct, surtout pour ce genre de travail dont le but essentiel reste l'obtention de couches épitaxiées dans la zone froide.

\section{Bibliographie}

[1] Klosse, K., Ullersma, P., J. Cryst. Growth 18 (1973) 167.

[2] Hofmeister, H. K., Haeseler, R. V., Glemser, O., Elektrochemie 64 (1960) 513.

[3] Potier, A., Colloque annuel du GFCC, Montpellier, févr. 1970

[4] Chevrier, J., Etienne, D., Camassel, J., Auvergne, D., Mat. Res. Bull. 7 (1972) 1485.

[5] Phalippou, J., Mazel, A. L., Thèse de $3^{\mathrm{e}}$ cycle, Acad. Montpellier, févr. 1970.

[6] Hurle, D. T. J., Mullin, J. B., J. Phys. Chem. Solids, Sup. no 1 (1967) 241.

[7] Berkowitz, J., Chupka, W. A., J. Chem. Phys. 48 (1968) 5743.

[8] Fujisaki, H., Westmore, J. B., Tickner, A. W., Can. J. Chem. 44 (1966) 3063.

[9] Goldfinger, P., Jeunehomme, M., Trans. Faraday Soc. 59 (1963) 2851

[10] Sedwick, T. O., Agule, B. J., J. Electrochem. Soc. 113 (1966) 54.

[11] Wösten, W. J., Geers, M. G., J. Phys. Chem. 66 (1962) 1252.

[12] Korneeva, V., Novoselava, A. V., Sokolov, V. V., Russ. J. Inorg. Chem. 5 (1960) 117.

[13] Yiм, W. M., Stoғko, E. J., J. Electrochem. Soc. 119 (1972) 381.

[14] Boev, E. I., Benderskit, L. A., Mil'kov, G. A., Russ. J. Phys. Chem. 43 (1969) 776.
[15] Charlot, C., Tikhomiroff, N., Laffite, M., Bull. Soc. Chem. France 2 (1970) 459.

[16] Lilley, P., Jones, P. L., Litting, N. W., J. Cryst. Growth 13-14 (1972) 371.

[17] Lilley, P., Jones, P. L., Litting, N. W., J. Mat. Sciences 5 (1970) 891.

[18] Stull, D. R., Sinke, G. C., Adv. Chem. (1956) no 18.

[19] Kelley, K. K., King, E. G., Contributions to the data on theoretical Metallurgy, XIV, Bull. 592, US Bur. Mines (1961).

[20] Kelley, K. K., KING, E. G., Contributions to the data on theoretical Metallurgy, XIII, Bull. 584, US Bur. Mines (1960).

[21] Lumbroso, H., "Nouveau traité de chimie minérale», P. Pascal, XIII (1961).

[22] Rawling, J. R., Toguri, J. M., Can. J. Chem. 44 (1966) 451.

[23] Gunn, S. R., J. Phys. Chem. 68 (1964) 949.

[24] SiRTL, E., Z. Naturforsch. 21a (1966) 2001.

[25] Sneed, M. C., Brasted, R. C., Comprehensive Inorganic Chemistry (New York) 1965, 112.

[26] Kuznztsov, F. A., Beyli, V. I., J. Electrochem. Soc. 117 (1970) 785

[27] Hirschfelder, J., Curtiss, C., Bird, R. B., Molecular Theory of gases and liquids (Wiley, New York) 1954.

[28] WilKe, C. R., LeE, C. Y., Ind. Eng. Chem. 47 (1955) 1253. 